\title{
Fast and revenue-oriented protection of radial LV cables with smart battery operation
}

\author{
Sara Ramezani*, Nicolas Höning* and Han La Poutré*† \\ *Centrum Wiskunde en Informatica, Science Park 123, 1098 XG Amsterdam, The Netherlands \\ ${ }^{\dagger}$ Utrecht University, Princetonlaan 4, 3511 CC Utrecht, The Netherlands
}

\begin{abstract}
Low-voltage radial electricity cables will have more and more difficulties to carry the increasing load of novel consumption devices (e.g. electric vehicles) and the expected generated input of decentrally-generated power (e.g. from photovoltaic cells). One solution to avoid replacement is to install a battery at the end of a cable which is expected to be overloaded frequently. The intelligent operation of this battery needs to combine the protection of the cable with optimizing its revenue, in order to be economically viable. This paper formulates the offline optimization problem and proposes two robust heuristic online strategies. We show in computer simulations that these heuristics, which make fast just-in-time responses, reliably deliver good results. Our second heuristic, $H_{2}$, reaches up to $83 \%$ of the approximated theoretical optimum.
\end{abstract}

\section{INTRODUCTION}

The future technology mix for electricity generation as well as consumption will put more powerful devices on the lower voltage levels of our grids. There, on the street level, radial cables that were designed for common household devices will soon have to carry the current that big consumption devices like electric vehicles and heat pumps demand, as well as the locally generated electricity (e.g. from photovoltaic cells). It is crucial that our energy infrastructure can support the transition to a modern energy future, but major updates are very expensive.

Too much aggregated load can surpass the maximum capacity of some segments of the cable. Of course, there can also be too much generation on the cable. Both cases lead to the overheating of the cable, which shortens its lifetime considerably. Facing increasing overloading situations of an LV cable, one obvious solution is to replace it with a larger cable. However, that is expensive and cannot happen in all streets at the same time due to budget constraints.

When envisioning an adaptive solution to the problem, a crucial issue is that the problem cannot be solved from the substation by increasing or decreasing voltage. Only nodes behind an overloaded segment are part of the problem and only they can contribute to a solution. Assuming there are smart meters, the cable operator can use the sensor data to deduce which segments are overloaded and to what degree.

Incentive mechanisms for residents are not a viable solution for this problem, since they would need to pay the originators of the problem to reverse it, resulting in strategic difficulties. Therefore, this paper proposes that the network operator can prolong the operation time of the cable by installing a smart battery at its end. This battery can react to most overload situ- ations by charging or discharging, thereby protecting the cable (a more thorough explanation will be given in Section III). As a battery is also expensive, it is important to consider that it can make profits during non-critical times, by buying and selling electricity, and thus (partly) pay for itself. To our knowledge, this is the first work that studies this kind of solution.

Employing such batteries on LV cables would not only have the local effect of cable protection, but also contribute to the global challenge of continuously balancing supply and demand in the whole electricity grid ${ }^{1}$.

Because cable protection and revenue maximization should be optimized together, we are dealing with multiple objectives. Here, expectations of future conditions are important. A strategy which is used to control the battery could consider expected overloading situations in future time slots, in order to be prepared to protect the cable (if protection involves charging, the battery should not be full, if protection involves discharging, the battery should not be empty). At the same time, expectations of energy prices influence the revenue that the battery can achieve.

This work advances the state of the art in the following ways: We first formulate the optimization problem for the battery. Given a global market price and the state of the cable, the battery needs to decide how much to buy or sell (in each time step). We present a deterministic offline solution to this problem as a linear program. Then, this paper begins to tackle this problem by proposing a suite of stylized heuristic online strategies that are reliable and compute actions online. We simulate the offline solution and the online strategies in experimental scenarios.

Results show that these heuristics reliably deliver good results. Our second heuristic $\left(\mathrm{H}_{2}\right)$ reaches $83 \%$ when compared to an offline solution which knows all prices in advance.

\section{BACKGROUND}

The notion that Computational Intelligence is needed to optimize the highly complex settings that can be expected in smart grids is gaining more and more traction (for an overview, see for instance Jiang et al (2009) [3]). The most interesting results can be expected when the interplay between several complementary technology aspects is highlighted.

\footnotetext{
${ }^{1} \mathrm{~A}$ further possible objective is to support islanding mode, but that would probably require a very large battery. In this work, we use a cost-efficient solution for the problem at hand, which is to be able to support the local network.
} 
Electricity storage (i.e. batteries) is a technology which complements many other technologies that are considered primary drivers in smart energy systems. One example is how batteries can buffer the output of intermittent renewable energy generation, a topic which has (mostly for large-scale batteries) received a lot of attention in the last decade (e.g. [5], [1]). The operation of batteries on lower levels of the grid has only recently begun to attract attention. A notable application are electric vehicles (EVs), which are expected to represent higher shares of the car fleet in upcoming years. Most work in this field has discussed decentralized mechanisms of scheduling the charging of fleets of EVs (e.g. [8], [2]). Furthermore, Vytelingum et al (2010) have studied the effect of large-scale penetration of batteries for smart home management on the overall grid [9].

This work looks into the interplay of storage with another important technological problem. Only recently have electrical engineers begun to pay attention to the fact that the increased expected activity on low voltage levels (by intermittent decentralized generation, i.e. by photovoltaics installed on roofs, and by increasing demand for electricity, e.g. by heat pumps or electric vehicles) threatens the operation of cables which were not designed for this usage [7].

Kadurek et al. (2011) [4] describe this challenge for the operation of low voltage cables in more detail, highlighting that conventional protection schemes will not be able to tackle overloading. They note that different segments of a cable can be in different states and that only a more sophisticated measuring infrastructure (e.g. by smart meters) can allow the Distribution System operator to identify the critical segments and in which state they exactly are. They thus make the case for a novel use case for sensory data, in which intelligent actions based on these data are of high societal benefit (because the lifetime of an expensive underground cable can be prolonged). Their proposed protection scheme operates in two phases, where the first phase assumes some (to be further determined) method of preventive action and the second phase involves protection (disconnection of customers). We concentrate on the preventive action in this work, for which until now no solution has been proposed (to the best of our knowledge).

There is currently no consensus among engineering experts about a standard cost function that represents economic losses experienced by overheating a cable. However, it is commonly agreed that some are more realistic than others. On the other hand, there are good reasons to model cost functions with stylized mathematical formulation, since the complexity of both devising robust algorithms as well as computing optimal offline solutions increases rapidly when more realistic cost functions are being used.

The strategy for the operation of the battery optimizes its behavior based on local observations of the cable state and the market prices. If exact prices are only known as they are announced, we face a so-called online problem. Here, information about the future is imprecise and the decision maker does not have much time to compute his best decision for time step $t$ (in smart grid settings, fast decision-making is of interest as the speed at which optimizing decisions need to be made increases). Heuristic strategies [6] are a good fit for online decision problems. They trade in accuracy for speed and can be used when exhaustive search is impractical.

\section{MODEL}

Time and energy price. Assume a finite number of time steps/units $t=1,2, \ldots, T$. The price of a unit of energy in each time step is determined by the grid and is $p_{t}$ at time $t$.

Battery. We have a battery with capacity $B$ that can be charged at a rate of at most $B_{+}$or discharged at a rate of at most $B_{-}$units of energy in each time unit. The battery can either be charged or discharged in each time step. We assume that the amount with which the battery is charged in time $t$ is $c_{t}$, and the amount it is discharged is $d_{t}$, where $c_{t}, d_{t} \in \mathbb{R}_{0}^{+}$. So at time $t$, the battery is charged $c_{t}$ units if $c_{t}>0$ and discharged $d_{t}$ units if $d_{t}>0$.

The battery also has an efficiency factor $\alpha$; so for every unit it is charged, it can only discharge $\alpha$ units of energy where $\alpha \in[0,1]$. Note that a solution where both $c_{t}$ and $d_{t}$ are positive will always have less revenue than one where $c_{t}-d_{t}$ units of energy are charged or $d_{t}-c_{t}$ are discharged (depending on which one among $c_{t}$ and $d_{t}$ is larger). This is because a factor of $\alpha$ of the energy is lost by storing it on the battery and then discharging. So when considering solutions, we can always assume that at least one of $c_{t}$ or $d_{t}$ is zero.

Finally, we designate by $b_{t}$ the level of charge in the battery in the beginning of time step $t$. We also assume that the loss of efficiency (by rate $\alpha$ ) happens when storing the energy on the battery.

Cable. There is also a cable with a sequence of consuming and producing agents. The maximum power capacity of the cable is $C \in \mathbb{R}^{+}$. The cable is connected to the grid. The battery is located at the end of the cable and all the agents are in between. The cable is divided into a number of segments, each segment between two of the consuming/producing agents, or the first agent and the substation, or the last agent and the battery. See Figure 1 for an example of how the cable is divided into segments. Each segment on the cable can have a different amount of power in each time step (see Figure 2).

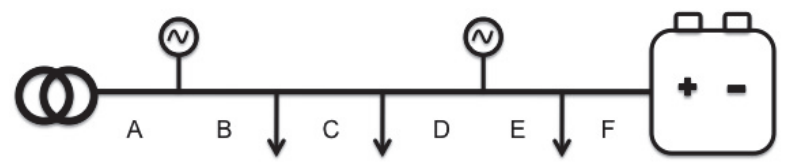

Fig. 1. The cable is divided into segments A-F.

We suppose that power flow in the cable in the direction of the battery is represented using positive real numbers and flow in the opposite direction is represented with negative numbers. So in any time step, an agent that uses a unit of energy will increase, and an agent that produces a unit of energy will decrease the flow of all the segments located before it by one unit. In Figure 2, an example of a number of producing and 
consuming agents and the power flow that they generate is illustrated. Similarly, if the battery charges a unit of energy, there will be an increase of one unit in all the segments of the cable and if it discharges, there will be a decrease of one unit in all segments.

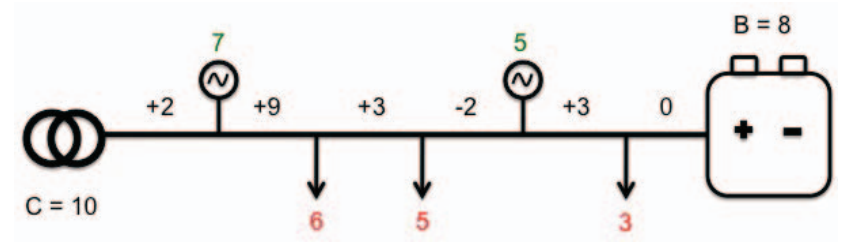

Fig. 2. Example problem when in a non-critical time step.

Capacity $C$ of the cable means that if the power flow of a segment is $e$ such that $|e|>C$, the cable will be overloaded. Thus, the flow in every segment in every time step is not necessarily relevant to the overloading problem; what is relevant is the highest and lowest amount of load among all of the segments (here we are talking about the flow that the agents cause, not including the battery). In each time step $t$, we will call the flow of the segment with the lowest flow $f_{t}^{-}$ and the flow of the segment with the highest flow $f_{t}^{+}$.

Critical time steps. If $f_{t}^{+}>C$ or $f_{t}^{-}<-C$ in a time step $t$, then $t$ is an overloaded time step or a critical time step. The amount of overload is then $\max \left(f_{t}^{+}-C,-f_{t}^{-}-C\right)$. We call any segment that has more than $C$ flow in any direction a critical segment or overloaded segment.

The battery may be able to "resolve" a critical time step by charging when the critical segments have negative flow and discharging when the critical segments have positive flow. By "resolving", we mean that the charging or discharging of the battery results in a configuration that is not critical any more. See Figures 3 and 4 for examples of critical time steps resulting, respectively, from excess consumption and production, and how the battery can resolve them.
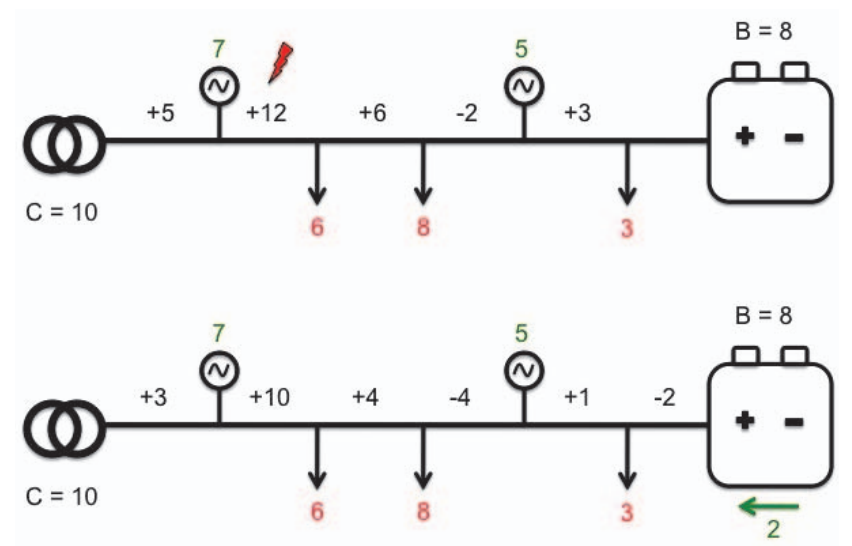

Fig. 3. Top: critical time step arising from excess consumption. Bottom: battery discharges 2 units of energy to resolve critical time step.

Note that it is not possible to completely resolve a critical time step using a battery in all situations, no matter how much
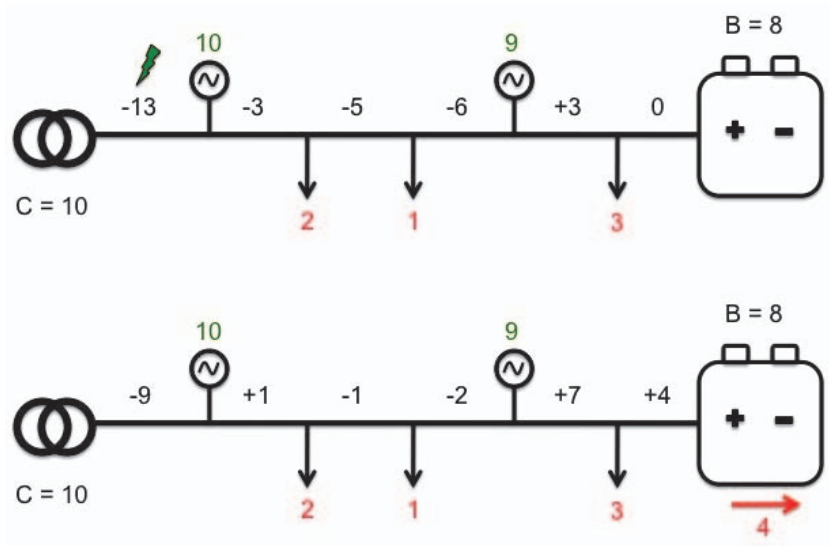

Fig. 4. Top: critical time step arising from excess production. Bottom: Battery charges 4 units of energy to resolve critical time step.

charge or capacity the battery has. It is not possible to resolve the crisis if there are both critical segments with a positive flow and critical segments with a negative flow, since resolving one will intensify the other. It is also not possible to resolve a critical time step if the amount of overload is more than the cable capacity, or more than what would result in an overload in the opposite direction.

Other than the above cases, where it is never feasible to use a battery to resolve a critical interval, it also may not be feasible to resolve some of the other critical intervals. This may be due to, for instance, restrictions on the battery (total capacity, charge/discharge capacity) or consecutive critical intervals (with no chance to recharge (or discharge) the battery in between). While a single battery may not be able to solve these extreme situations, it can be useful in many of the ordinary critical time steps that occur more often. Also, the battery may not be able to resolve the critical interval completely in some of these cases, but it can reduce the overload and protect a cable that might otherwise be damaged beyond repair. Furthermore, advances in the capacity, charging rate, and efficiency of batteries can lead to batteries that can cover even more cases.

Cost function. We want to be able to evaluate the amount of damage that a critical interval causes. In order to do this, we suppose that there is a cost function that assigns a value to each time step based on how much the cable was overloaded (potentially damaged) in that time step, $v: \mathbb{R}^{+} \times \mathbb{N} \cup\{0\} \rightarrow$ $\mathbb{R}$. The first variable of this function is the magnitude of the maximum flow on the cable and the second shows the order of the time step in the set of consecutive critical time steps that contain it (it is $k$ for the $k^{\text {th }}$ consecutive critical interval). So, $v(x, k)$ is the cost of excess overload for a time step when the maximum flow on a segment of the cable is $x$ and the time step is the $k^{\text {th }}$ consecutive overloaded time step.

We assume that the same magnitude of overload in either direction has the same kind of negative effect, so the first variable is a positive real number. For a non-critical time step, the cost is always zero. Also, $v$ should be a non-decreasing 
function on each of the variables. Our primary aim is to minimize the overall damage to the cable, i.e. sum of the cost over all time steps, $V$.

Cost functions can be designed to be more stylized or realistic. Either one or both the magnitude of the overload and the consecutiveness factor can be disregarded in more stylized functions. For instance a simple cost function can assign a unit of cost to each overloaded time step. Minimizing the cost based on such a cost function results in a solution that resolves as many critical time steps as possible.

In real-world overload problems, however, the temperature that the cable reaches has an important effect on the amount of damage to it. So more complicated functions that depend on the magnitude of the overload may be more realistic. A basic example is a cost function that assigns the magnitude of overload to each overloaded time step:

$$
v_{\operatorname{mag}}(x, k)= \begin{cases}x-C & x>C \\ 0 & \text { otherwise. }\end{cases}
$$

It is even more realistic to use functions that take into consideration the fact that consecutive overloaded intervals are more damaging to the cable, and assign extra costs to them. In this work we consider such a function as the cost function:

$$
v(x, k)= \begin{cases}c_{h}^{k} & x>C \\ 0 & \text { otherwise }\end{cases}
$$

where $c_{h}$ is a constant (larger than 1). Setting $c_{h}>=1$ guarantees that in a consecutive set of critical time steps, each time step becomes more costly than the next.

Finally, we need to balance a trade-off between reducing the cost of overloading the cable and maximizing the revenue of the battery operator. We consider a general formula for combining the two objectives and giving them weights; so we aim to minimize $\omega V-R$. Here $R$ is the total revenue, $V$ is total cost added over all time steps, and $\omega$ is a constant weight. By adjusting $\omega$, we can prioritize between the two objectives. For example, if $\omega \rightarrow \infty$, the solution with the highest revenue is selected among those that have the lowest cost. We shall usually consider a case where $w$ is relatively large, because protecting the cable should be a higher priority for the battery operator.

\section{A. Offline Optimization Problem}

The offline optimization solution aims to find $c_{1}, \ldots, c_{T}$ and $d_{1}, \ldots, d_{T}$, such as to minimize $\omega V-R$, given that exact values of $p_{t}, f_{t}^{+}$, and $f_{t}^{-}$are known throughout the time horizon. It can be formulated as:

$$
\min _{c_{1}, \ldots, c_{T}, d_{1}, \ldots, d_{T}} \omega V-R=\omega \sum_{t=1}^{T} v\left(x_{t}, k_{t}\right)-\sum_{t=1}^{T} p_{t}\left(d_{t}-c_{t}\right)
$$

such that $\forall t \in\{1, \ldots, T\}$ :

$$
\begin{aligned}
& 0 \leq c_{t} \leq B_{+}, \quad 0 \leq d_{t} \leq B_{-} \\
& 0 \leq b_{1}+\sum_{1}^{t}\left(\alpha c_{t}-d_{t}\right) \leq B
\end{aligned}
$$

where $x_{i}=\max \left(\left|f_{i}^{+}+c_{i}-d_{i}\right|,\left|-f_{i}^{-}-c_{i}+d_{i}\right|\right)$, i.e. the maximum amount of flow, and $k_{t}=i$ s.t. $x_{t-i} \leq C \wedge \forall j=$ $0, \ldots, i-1, x_{t-j}>C$.

Note that because of the exponential factor in the $V$ function and the structure of $k_{t}$, this function is very complicated to compute analytically. However we can formulate an LP to approximate the optimal solution to this problem, assuming that there is a solution with at most a constant $k$ consecutive critical time steps (if it is actually so, the LP will in fact result in the exact optimal solution). In order to obtain this LP, we reformulate the optimization problem as follows:

$$
\min _{c_{1}, \ldots, c_{T}, d_{1}, \ldots, d_{T}} \omega \sum_{t=1}^{T} v_{t}-\sum_{t=1}^{T} p_{t}\left(d_{t}-c_{t}\right)
$$

such that $\forall t \in\{1, \ldots, T\} \wedge \forall k \in\{1 \ldots t\}$ :

$$
\begin{gathered}
0 \leq c_{t} \leq B_{+}, \quad 0 \leq d_{t} \leq B_{-} \\
0 \leq b_{1}+\sum_{1}^{t}\left(\alpha c_{t}-d_{t}\right) \leq B \\
v_{t} \geq e_{i} \times C O_{t}^{k} \\
C O_{t}^{k} \in\{0,1\} \\
C O_{t}^{1} \geq\left(x_{t}-C\right) /(\operatorname{Max} x-C)
\end{gathered}
$$

and $\forall t \in\{2, \ldots, T\} \wedge \forall k \in\{2 \ldots t\}$ :

$$
C O_{t}^{k} \geq C O_{t-1}^{k-1}+C O_{t}^{k-1}-1.5
$$

Where $e_{k}=c_{h}^{k}$ and $\operatorname{Max} x$ is a constant number that is larger than all flows on the cable. Also, $v_{t}$ is a variable that represents $v\left(x_{t}, k_{t}\right)$ and $C O_{t}^{k}$ is an integer variable that specifies whether if $t$ is at least the $k^{\text {th }}$ consecutive overloaded time step; it is equal to 1 if it is and equal to zero otherwise.

\section{HEURISTICS}

In this section we present two heuristic strategies for solving the online problem. Both make two general assumptions about critical time steps, namely that their avoidance takes precedence (also over revenue optimization) and that resolving them as much as possible is worthwhile (even if the time step would still be critical). These assumptions make the algorithms robust, while their implementation remains straightforward and their computation time is low. They also make sure that the algorithms will never increase an overload, a property that does not necessarily hold for the solutions of given by the LP defined above.

\section{A. Robust Heuristic}

We define a basic heuristic, $H_{1}$, which is a robust algorithm to improve network stability and serves as a benchmark. Algorithm 1 describes $H_{1}$ in detail.

The basic idea of this heuristic algorithm is that if the current time step is a critical one, $H_{1}$ resolves it as much as is feasible given the battery charge level and charge and discharge rates. If the interval is not critical, it always tries to bring the charge in the battery to half of its capacity. 
The algorithm uses none of the expectations for prices and cable flows of the intervals after the current interval in its calculations. It does not even use the price of energy in the current interval. It always brings the charge in the battery to half of its capacity so that it could be prepared to resolve both critical intervals that call for charging and critical intervals that call for discharging as much as possible, assuming it will not know which one to expect.

In more detail, the first objective of $H_{1}$ is to always contribute to resolving critical time steps, as far as possible. However, a contribution in either direction is limited by the highest load in the opposite direction. So the battery can at the most contribute the mean of the distance between $f_{t}^{+}$and $f_{t}^{-}$. This is done in line 2. Lines 3 through 7 make sure that the contribution is only as high as necessary. In all non-critical time steps, the battery attempts to adjust its current charge level, $b_{t}$, towards half of its maximum charge level $\left(\frac{B}{2}\right)$, see line 9 . In line 11 , the efficiency factor of the battery $(\alpha$, see Section III) is taken into account when charging, in order to reach the target level. Lines 13 and 14 make sure that this action does not induce overloading.

Each chosen action is, of course, restricted by the maximal charging rates $B_{+}$and $B_{-}$(see line 16), the available space in the battery when charging (capacity $B$ minus level $b_{t}$, see lines 17 through 19) and the available level $b_{t}$ when discharging (see line 20).

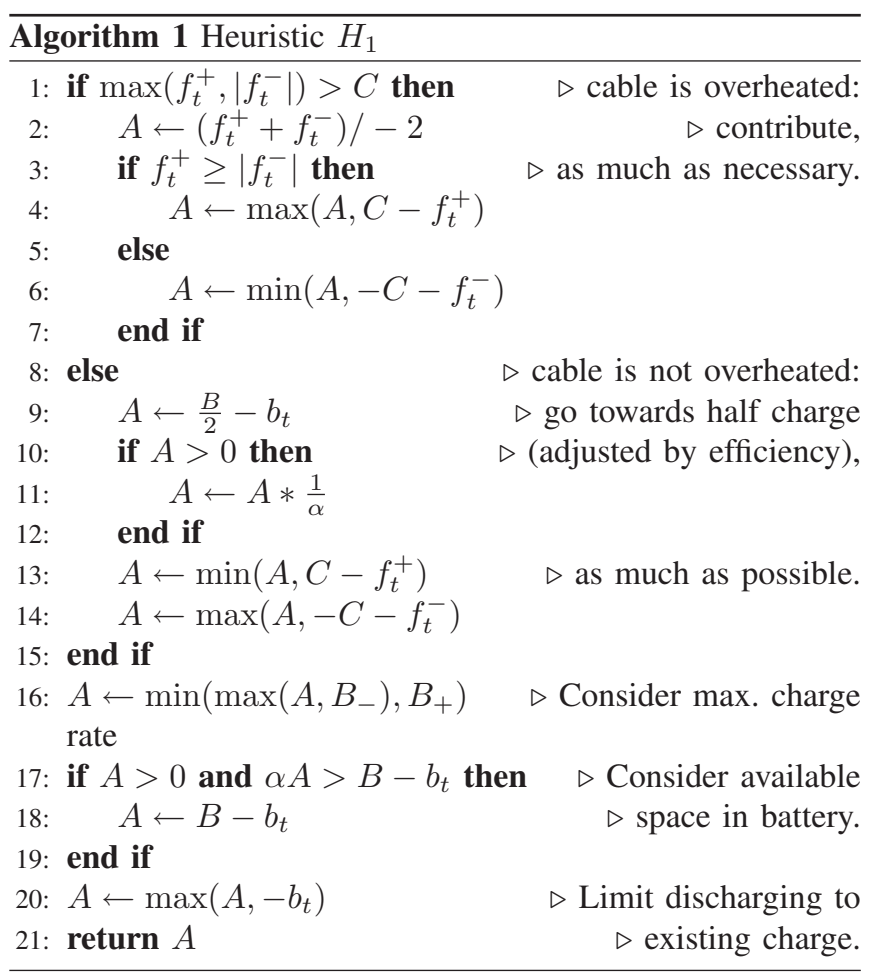

\section{B. Price-based Heuristic}

We propose a second heuristic, $\mathrm{H}_{2}$, which is robust (tries to resolve critical intervals) but also optimizes revenue. For this, it will use an expectation of prices in order to estimate the maximal and minimal level on the cable for the remaining time steps (without its own activity). It then proceeds in three stages:

1) Each future time step is classified into having a low or high (expected) price. Based on this, each time step is assigned a maximal buy- or sell-volume, respectively. If the interval is expected to be critical, the aim will be to resolve as much as possible given the cable constraints (in a similar manner as what happens in this case in $H_{1}$ ). Otherwise, if the interval is classified as high-price, the battery will try to sell as much as it can and if it is classified as low-price it will try to buy as much as possible.

Basically, in this step, we devise a list of what we wish to achieve in each time step given our estimated relative price and cable capacity, assuming that the battery has enough capacity. Thus, the maximal charging rates of the battery, $B_{+}$and $B_{-}$, are taken into account when computing the desired buy/sell volumes. However, the capacity of the battery itself is not enforced yet here because it cannot be computed independently of the intervals before and after it.

2) Now this preliminary schedule is made feasible, such as to keep the battery charge within its physical limitations $[0, B]$ in every time step. We do this while having the expected revenue in mind. Going forth from the current time, the non-critical time steps are merged into blocks based on whether we desire to sell or buy in them. If fulfilling the desire of all the time steps within a block would result in a overcharged or over-discharged battery, the desired amounts are adjusted to fall within the battery limits.

This means that if a block of time steps in which we prefer to buy will result in a overcharged battery, we will decrease from the buy-volume of the most highly priced time steps within the block. Similarly, if fulfilling a block of negative-wished time steps results in a battery that has a negative amount of charge, we will decrease from the sell-capacity of those that have the lowest price within the block.

At the end of this step we can be sure that we have a feasible solution if we set each $c_{i}-d_{i}$ to the desired amounts that we currently have (remember that one of $c_{i}$ and $d_{i}$ must be equal to zero in any solution that we consider, i.e. we never charge and discharge in the same time step).

3) Finally, in the last step we update the desired sell or buy values such that our expected critical intervals can be resolved as much as possible. This time the first expected block of same-sign critical time steps, $\beta$, is considered. By same-sign critical time steps we mean critical time steps for which the best desirable action is either to sell or to buy. The amount of charge needed in the battery in order to resolve all time steps in $\beta$ is computed. For 
instance, if $\beta$ calls for selling $x$ units of energy, the battery would need to be charged to at least $x$ units to fulfill that. If $x>B$, then we try to do as best as we can, which is to fill the battery to full capacity. Similarly if $\beta$ calls for buying $y$ units of energy, then we want to make sure that the battery is charged to at most $B-y$ before the block begins. Again, in the case that $y>B$ we try to achieve an empty battery.

Now, given the demand of $\beta$, we adjust the wished buy and sell volumes in the non-critical time steps before it once more. To do this, if the amount of previous wishes results in a battery with too much charge, then we decrease from the buying volume of the intervals in which we wish to buy (among those that come before $\beta$ ), starting from those with the highest price. The feasibility of decreasing from each such interval is checked and the next interval is considered if the result of decreasing is infeasible. Similarly, if fulfilling the previous set of wishes results in an undercharged battery, then we decrease from the selling volumes of the non-critical intervals, starting from those with the lowest price and checking feasibility while we go ahead.

This process is updated in each time step, using the actual price of that time step and the expected price of the rest. Thus the heuristic adapts its calculations using the situation that takes place after the actual price and energy production and consumption of that time step is known.

The advantage of $H_{2}$ compared to $H_{1}$ is that revenue considerations are taken into account as well as more precise and tailored preparations for critical time steps. For instance in steps 2) and 3), whenever we need to sell less we try to do that in intervals that have a lower price and whenever we want to buy less, we try to buy less in intervals with higher price, if feasible. The resulting algorithm is still a fast algorithm that works in polynomial time and can be executed in real time.

\section{EXPERIMENTS AND SIMULATIONS}

\section{A. Setup}

We now explain the setup of scenarios in which we evaluate the heuristics. Overall, we construct two scenarios; in each we systematically vary $\omega$, the weight of costs in the evaluation of the battery performance. The detailed settings can also be read from Table I. We made the code we use to run the simulations available online ${ }^{2}$.

\section{Network element specifications}

The cable settings used here are inspired by settings that are common in Europe, but most of these also apply to grids in other parts of the world. We assume an LV feeder that can carry a current of 200 Ampere $(I)$ and that the potential difference is 230 Volt $(V)$. The capacity for power $P$ is given by $P=I * V$, so we assume a value of $46 \mathrm{~kW}$ for the cable capacity $C$. On each of the three phases, 20 customers are equally distributed. We consider one phase on the feeder, and

\footnotetext{
${ }^{2}$ https://github.com/nhoening/battery-heuristics
}

thus the number of customers, $N$, is 20 , of which we model 10 as identical consumers and 10 as identical generators.

To model the connected customers, we base values on currently common settings, but also extrapolate to future settings with more devices (which could pose problems for LV cables). The maximal demand of a consumer household, $D^{\max }$, is assumed to be $4 \mathrm{kWh}$. In addition, we assume that consumers own electric vehicles, the batteries of which are being charged by up to $1 \mathrm{kWh}$, between $7 \mathrm{pm}$ and $7 \mathrm{am}$. The local supply $S^{\max }$ is $5 \mathrm{~kW}$, assuming that a common photovoltaic array of 20x 250W is installed ${ }^{3}$. In our scenarios, the PV cells produce electricity only during $12 \mathrm{am}$ and $4 \mathrm{pm}$, which we assume to be strong sunlight hours, but then constantly at maximum capacity (thus, in each of these hours they produce $5 \mathrm{kWh}$ ).

Furthermore, we assume that the battery capacity $B$ is $31 \mathrm{kWh}$, and the maximum charging rate $B_{+}$is $5 \mathrm{~kW}$. These values are taken from specifications of an EV battery currently on offer ${ }^{4}$. We also assume that the battery discharges as fast as it charges, so $B_{+}=B_{-}$, and that it has an efficiency of 0.8 (only $80 \%$ of charged electricity can be discharged due to conversion losses).

For the simplicity of our mechanism, we will in this work assume that power flows remain constant over the course of one time step. We also do not consider reactive power or losses by distributing power over distances. We also note that our model proceeds in half hour intervals ${ }^{5}$. This means that only half of the energy (of consumption or generation) that we have described in $\mathrm{kWh}$ will get delivered per time step in our model. For instance, in our model each solar panel produces only $2 \mathrm{kWh}$ of energy in each of the 16 half-hour time steps between $12 \mathrm{am}$ and $4 \mathrm{pm}$. However, the instantaneous power output at any given moment (given in $\mathrm{kW}$ ) remains unchanged, because $x \mathrm{~kW}$ of continuous instantaneous power output are required to produce $\frac{1}{2} x \mathrm{kWh}$ in only half an hour.

We model two ways of placing consumers and producers along the cable. The first option is that they are situated alternately along the cable, beginning with a consumer. This is the most optimistic setup for the magnitude of possible overloading. The other option is the most pessimistic one, meaning that all consumers are situated next to each other, followed by all generators, who are situated next to each other, as well.

\section{Economic assumptions about demand}

For realistic input of price series to model such price dynamics, we obtained half-hour spot market prices from the UK wholesale power market $^{6}$ for the first ten months of 2012. We removed weekend days from the data (leaving us with 219 days), in order to ease predictions for algorithms that have to work with expected prices. For price expectations,

\footnotetext{
${ }^{3}$ compare http://www.contemporaryenergy.co.uk/pv_products.htm

${ }^{4}$ see http://app.codaautomotive.com/CarConfigurator/View/Specifications

${ }^{5}$ This is because we use real-world price data from the UK wholesale market (see below) to make use of actual electricity price dynamics. A realistic setting from a technological standpoint would be 15 minutes, which we aim for in follow-up work.

${ }^{6} \mathrm{http}: / /$ www.apxendex.com/market-results/spot-markets/apx-power-uk/
} 


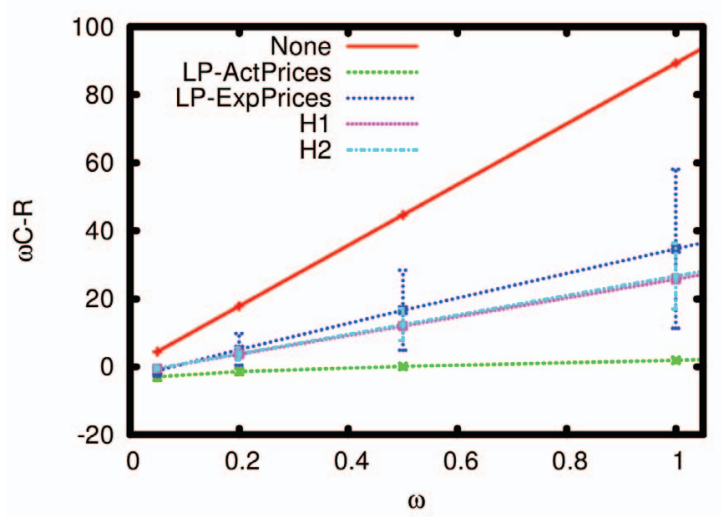

(a) optimistic

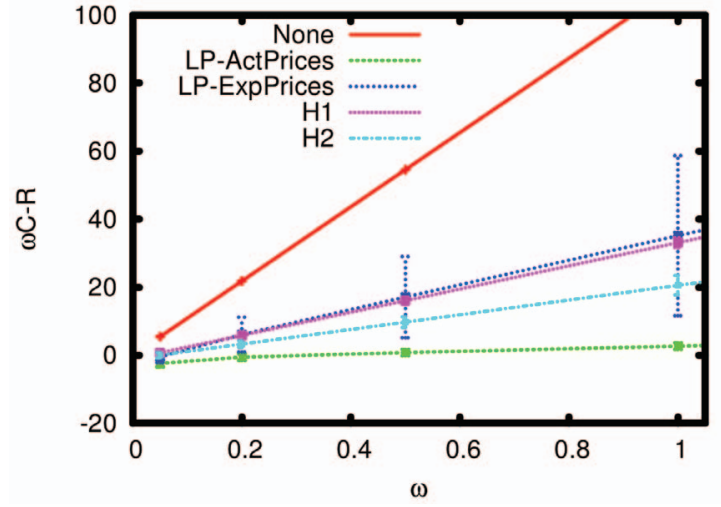

(b) pessimistic

Fig. 5. Simulation results for the two scenarios

we computed an average price profile for each day of the month. To make good predictions is not the focus of this work and such preparations seem reasonable. The wholesale market prices in this data set lie roughly in the range between 0.03 to $0.07 £ / \mathrm{kWh}$. We multiplied these wholesale prices by $3 \frac{1}{3}$ to arrive at a price range that reflects prices on retail markets. Our resulting prices lie roughly in the the range between 0.10 and $0.23 £ / \mathrm{kWh}$. Those prices are appropriate for the UK and Europe in general $^{7}$.

Consumers in our model are not adapting their behaviour based on the market price, but instead simply act according to their needs. They are assumed to act in accordance with the majority of other consumers in the market. Furthermore, we can assume that the aggregated behaviour of the majority of consumers in a market determines the market price $\rho_{t}$. Thus, the consumers in our model consume the most of their energy usage when $\rho_{t}$ is high and consume only little energy when $\rho_{t}$ is low. Consequently, their price elasticity ${ }^{8}$ is positive. The demand function $d$ is given below (where we assume that the maximal quantity $D^{\max }$ is bought at $\rho^{\max }$, the maximal price from our data set, i.e. $\left.d\left(\rho^{\max }\right)=D^{\max }\right)$.

$$
d\left(\rho_{t}\right)=\left(D^{\max }-\gamma \rho^{\max }\right)+\gamma \rho_{t}
$$

Scenarios We create two scenarios, one with optimistic placement of customers and one where placement is pessimistic. Each scenario is evaluated for the duration of one day, so $T=48$. We initially drew a random set of 20 daily price series from the whole set of UK power market price series, on which we run all scenarios. Furthermore, we evaluate five strategies in each scenario: The first strategy is to use no battery at all (None). Furthermore, we employ the optimal offline solution (see Section III) with either complete knowledge about future prices (LP-ActPrice) or expected prices (LP-ExpPrices). Finally, we test the two heuristic strategies proposed in Section IV, $H_{1}$ and $H_{2}$. All strategies that work

\footnotetext{
${ }^{7}$ compare http://www.parliament.uk/briefing-papers/SN04153.pdf

${ }^{8}$ Price elasticity describes the percentage change in quantity demanded in response to a one percent change in price.
}

TABLE I

DEFAULT SETTINGS

\begin{tabular}{|c|l|l|}
\hline Name & Definition & value(s) \\
\hline \hline$T$ & (half-hour) time steps & 48 \\
\hline$C$ & cable capacity & $46 \mathrm{~kW}$ \\
\hline$N$ & number of customers on cable & 20 \\
\hline$B$ & battery capacity & $31 \mathrm{kWh}$ \\
\hline$B^{+}, B_{-}$ & max. (dis)charging rates & $5 \mathrm{~kW}$ \\
\hline$\alpha$ & efficiency factor & 0.8 \\
\hline$\gamma$ & slope of demand functions & 0.5 \\
\hline$D^{\text {max }}$ & $\begin{array}{l}\text { maximal demand } \\
\text { per consumer per step }\end{array}$ & $5.0 \mathrm{kWh}(7 \mathrm{pm}$ to 7am) \\
\hline$S^{\text {max }}$ & maximal supply & $5 \mathrm{kWh}(12 \mathrm{am}$ to $4 \mathrm{pm})$ \\
\hline$\omega$ & weight of cost-consideration & $.05, .2, .5,1,2$ \\
\hline$c_{h}$ & $\begin{array}{l}\text { cost factor for consecutive } \\
\text { overheating }\end{array}$ & 1.2 \\
\hline
\end{tabular}

on expected prices have the ability to calculate $f_{t}^{+}$and $f_{t}^{-}$ when given a price $p_{t}$ (that is, they are equipped with a model of locations and behaviours of the customers on the street).

The series of expected prices (which is used by the offline strategy $L P$-ExpPrices and the heuristic strategy $H_{2}$ ) is the average price series from all days of the month in which the current day lies. Furthermore, let $\rho_{m}$ denote the mean price per $\mathrm{kWh}$ of the month in which the current day lies. We assume the battery to be charged $\frac{B}{2} k W h$ before the day begins, for which we subtract $\rho_{m} * \frac{B}{2} £$ from its account. At the end of each day, we add to its account $\rho_{m}$ times the number of $\mathrm{kWh}$ of electricity left in the battery. In each scenario, we vary $\omega$, which denotes the weight with which we multiply overheating costs in the overall revenue function of the battery.

The linear program that represents the offline strategies is calculated by the GNU Linear Programming kit ${ }^{9}$. Because of the long computation time and the high number of evaluated settings, we limited the running time of the linear programs to fifteen minutes ${ }^{10}$. We set the highest expected number of consecutive critical time steps $(k)$ to ten.

\footnotetext{
${ }^{9}$ http://www.gnu.org/software/glpk/

${ }^{10}$ We ran the linear programs on selected settings for one hour and found that they achieve comparable performance.
} 


\section{B. Results}

General remarks Overall, all strategies lower the costs on an LV cable that often is overloaded by more than $50 \%$. An increase of $\omega$ leads to an increase in costs, which could of course be expected. However, $\omega$ does not have an influence on the ranking between strategies, which shows us that the outcomes of our model do not depend on how high overheating costs are in comparison to revenues made by the battery.

Comparison of strategies In both scenarios, LP-ActPrice performs best, as it has advance knowledge about actual prices. However, also our heuristics $H_{1}$ and $H_{2}$ can significantly reduce costs. Suppose that the distance between the performance of None and LP-ActPrice denotes $100 \%$, then $\mathrm{H}_{2}$ reaches $83 \%$ of the approximated theoretical optimum in the pessimistic scenario and both $H_{1}$ and $H_{2}$ reach $66 \%$ of the approximated theoretical optimum in the optimistic scenario. $H_{2}$ performs better than $H_{1}$ in the pessimistic scenario, while the differences between the two are not significant in the optimistic scenario.

The performance of $H_{1}$ is remarkably stable, with very low variance in outcomes. This is due to the non-speculative and robust nature of the algorithm. LP-ExpPrice always performs worst and its performance also varies the most by far. This is because the algorithm does not use online information and computes its schedule beforehand.

\section{CONCLUSIONS AND FUTURE WORK}

This paper discusses the strategy design problem for operating a battery at the end of a radial low voltage cable that is frequently overloaded. We formulate the offline optimisation problem as well as two robust heuristic online strategies. We show good performance of the heuristics in computer simulations, where our second heuristic $\mathrm{H}_{2}$ reaches up to $83 \%$ of the (approximated) theoretical optimum.

There are several opportunities for future work. First, the underlying cost function could be made more realistic (but also mathematically more challenging). It could consider each segment on the cable separately and also take the magnitude of overloading into account. It could also be interesting to add the objective of improving voltage conditions to the problem and to conduct lab experiments with a real LV cable. Furthermore, it could be interesting to develop a near-optimal online solution as a benchmark, which re-computes the expected returns of each possible action at every time step. Finally, it would be interesting to add a scenario with price-responsive consumers (representing so-called "active demand"), because their presence migh impair the battery's ability to profit from differences in prices.

\section{ACKNOWLEDGMENT}

The authors would like to thank Petr Kadurek for helpful comments.

\section{REFERENCES}

[1] L. Exarchakos, M. Leach, and G. Exarchakos. Modelling electricity storage systems management under the influence of demandside management programmes. International Journal of Energy Research, 33(1):62-76, 2009.

[2] E. H. Gerding, V. Robu, A. Rogers, and S. Stein. Online Mechanism Design for Electric Vehicle Charging. In Proceedings of the 10th International Conference on Autonomous Agents and Multiagent Systems (AAMAS), pages 811-818, 2011.

[3] Z. Jiang. Computational Intelligence Techniques for a Smart Electric Grid of the Future. In 6th International Symposium on Neural Networks, pages 1191 - 1201, 2009.

[4] P. Kadurek, J. Cobben, and W. Kling. Overloading Protection of Future Low Voltage Distribution Networks. In Proceedings of the PowerTech Trondheim Conference, 2011.

[5] M. Korpaas, A. Holen, and R. Hildrum. Operation and sizing of energy storage for wind power plants in a market system. International Journal of Electrical Power \& Energy Systems, 25(8), 2003.

[6] Z. Michalewicz and D. Fogel. How to solve it: modern heuristics. Springer, 2004

[7] P. Trichakis. Predicting the technical impacts of high levels of smallscale embedded generators on low-voltage networks. Renewable Power Generation, 2(4):249 - 262, 2008.

[8] S. Vandael, N. Boucké, T. Holvoet, K. De Craemer, and G. Deconinck. Decentralized coordination of plug-in hybrid vehicles for imbalance reduction in a Smart Grid. In International Conference on Autonomous Agents And Multiagent Systems, 2011.

[9] P. Vytelingum, T. Voice, S. Ramchurn, A. Rogers, and N. Jennings. Agent-based Micro-Storage Management for the Smart Grid. In Proceedings of the 10th International Conference on Autonomous Agents and Multiagent Systems (AAMAS), pages 39-46, 2010.

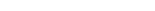

